\title{
How to connect a sensor to the Internet
}

Dipl.Ing. Thomas Schildknecht, Schildknecht AG, 71711 Murr, thomas.schildknecht@schildknecht.ag

\section{Summary}

The task of connecting sensors to the Cloud sounds trivial at first. It must be clarified whether a sensor is located within a company network (vertical network) or outside (horizontal network). Within a company network, it is quite simple, it can be very expensive if you cannot access an Internet access infrastructure such as Ethernet \& WLAN for technical and / or organizational reasons. The lecture describes which organizational requirements can be found in practice and which technical methods have been proven so far.

\section{Mega trends Cloud, Internet of things (IoT) and Industry 4.0}

The buzzwords stated above stand for the trend of digitization. Within a corporate network digitization is nothing really new and has been sate of the art for many years. Remote maintenance for facilities that are not local is also being used successfully. This application reduces travel times and costs, since in the event of an error access via a so-called VPN (Virtual Private Network) connection is possible. However, VPN connections are reaching their limits when it comes to continuously transferring data from a large number of devices. If these devices are also distributed worldwide, e.g. in an industrial application with pumps, VPN is not scalable and very expensive. Another hurdle is the availability of Internet access technology at on site. Even if a network with Internet access is available to an end customer using the pump, there is little chance that a supplier could also use it to connect its sensor. The security requirements of companies usually do not allow this. This means that in the context of this lecture alternative Internet access technologies will be discussed for these kind of tasks.

\section{Connectivity}

\subsection{LoRA, Sigfox \& Cellular Radio 2G-4G}

Possible Internet access technologies are LoRa, Sigfox and cellular radio, to name a few. LoRA and Sigfox are only available in a few places. Sigfox relies on a provider to operate a base station on site. LoRA also uses the royalty SRD band at $868 \mathrm{MHZ}$, but anyone can operate a base station. The range is in practice between max. 300 to max. $1 \mathrm{~km}$, depending on the antenna of the sensors. The technology aims at very cost-effective data transfer of a few euros per year. Data can only be transferred in small packages of a few bytes per day.
Cellular Radio (2G-4G) are available throughout the world. Using this technology, one has to rely on a contract with a mobile service provider who provides and maintains the network and sets a fee for it. Cellular phone contracts have not been offered to date for IoT device requirements. In international deployment, there are also completely new tasks. Roaming functionalities are limited for SIM cards meaning that they are switched off automatically after a certain. Furthermore, roaming partners in the country of use are not guaranteed including network coverage at the site. Previous contracts for M2M (machine to machine) communication are also limited by the data volume and are usually more expensive than end customer contracts. Thus, the industrial user must know at the time of delivery in which country the system will be used later. So far, these limitation limit successful usage of cellular radio technology. The lecture describes a new generation of M2M - IoT SIM cards and contracts with many new possibilities such as eSIM, unseteered roaming and role-based management.

\subsection{Cloud Portal}

In order to send data from a sensor to the Internet, you need a data storage with an IP address that can be reached worldwide and a software that handles the many different involved. This is called a cloud or data portal.

Currently, more than 700 data portals are available on the market. Data portals from the best-known providers are SAP HANA, Microsoft Azure, Amazon Iot, IBM Siemens Mindsphere, Software AG Cumolocity etc. These portals are almost exclusively data portals that store, process and visualize data. They can be operated within the company network or hosted publicly in a professional data center. In contrast, there are a few device portals that can handle the management of the connected devices, update the operating system over-the-air (OTA), and coordinate users' access rights to devices, etc. One of the most well-known device Portals is iCloud from Apple. Here, the connected "devices" are managed by Apple. This includes the update management of the operating system 
and the programs (APPs). This function is also required for IoT devices. The data cloud and the device cloud exchange data through a standardized software interface, the RESTful API.

\subsection{Interfaces to the process}

Typical data sources in industrial applications are sensors or machines and their control units. These have partially completely different hardware and software interfaces. These range from 0-10V / 4-20mA analog sensor signals, SPI, RS232 / 485 to fieldbus connections using Profibus, Profinet, or Powerlink etc. Also wireless sensor connections like Enocean, 802.15.4 (6LlowPan), Wifi or Bluetooth Low Energy is already available on the market. These interfaces and protocols are not suitable for data transmission to a data cloud. For this reason, gateways (another term is IoT Edge Gateway) are needed.

\subsection{IoT Edge Gateway}

Gateways take over the interfaces and protocol conversion from the sensor to the data cloud. Mostly they are stand-alone devices, as this functionality cannot be directly integrated into a sensor (yet). When using one or more wireless interfaces (e.g., Bluetooth for sensor interfacing and cellular for worldwide connectivity), it needs to be considered that every radio system in Europe needs to be certified according to RED (Radio Equipment Directive). Often, this procedure is not economically feasible for sensors as well as time consuming.

IoT edge gateways have a pre-processing functionality, which reduces the received sensor data volume according to application-specific needs. This is needed in order to minimize costs for the data transmission or to increase the service life of the battery during battery operation. In addition to the operating system of the gateway, it must also be possible to transfer new applications such as the rules for pre-processing or the measurement intervals to the gateway. This should preferably be done over-the-air (OTA), so persons are needed locally.

\subsubsection{Integration of an IoT Gateway}

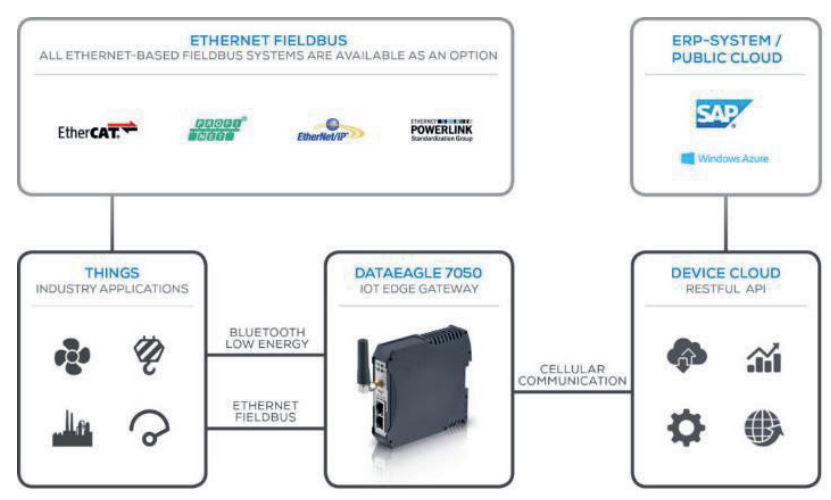

Picture 1 From, ,Thing“ to Cloud

\section{Conclusion}

With horizontally connected data networks a variety of new tasks, from process knowhow, selection of suitable sensors, the connection to control units, connectivity, IT protocols within the Internet communication, complex security requirements up to the contract design with the mobile radio providers arise. Thus, the goal is to integrate all these functions into a gateway and device cloud, that provide standard interfaces to the sensor and to many data clouds through the device cloud. 